\title{
Impact of Cardiac Magnetic Resonance Imaging
}

- Cardiac Contusion With Intramural Hemorrhage -

Aidan J.C. Burrell, MD; James L. Hare, PhD; Prabath J. Francis, MD;

Mark Fitzgerald, MD; David J. Cooper, MD; Deirdre Murphy, MD;

David M. Kaye, PhD; Andrew J. Taylor, PhD

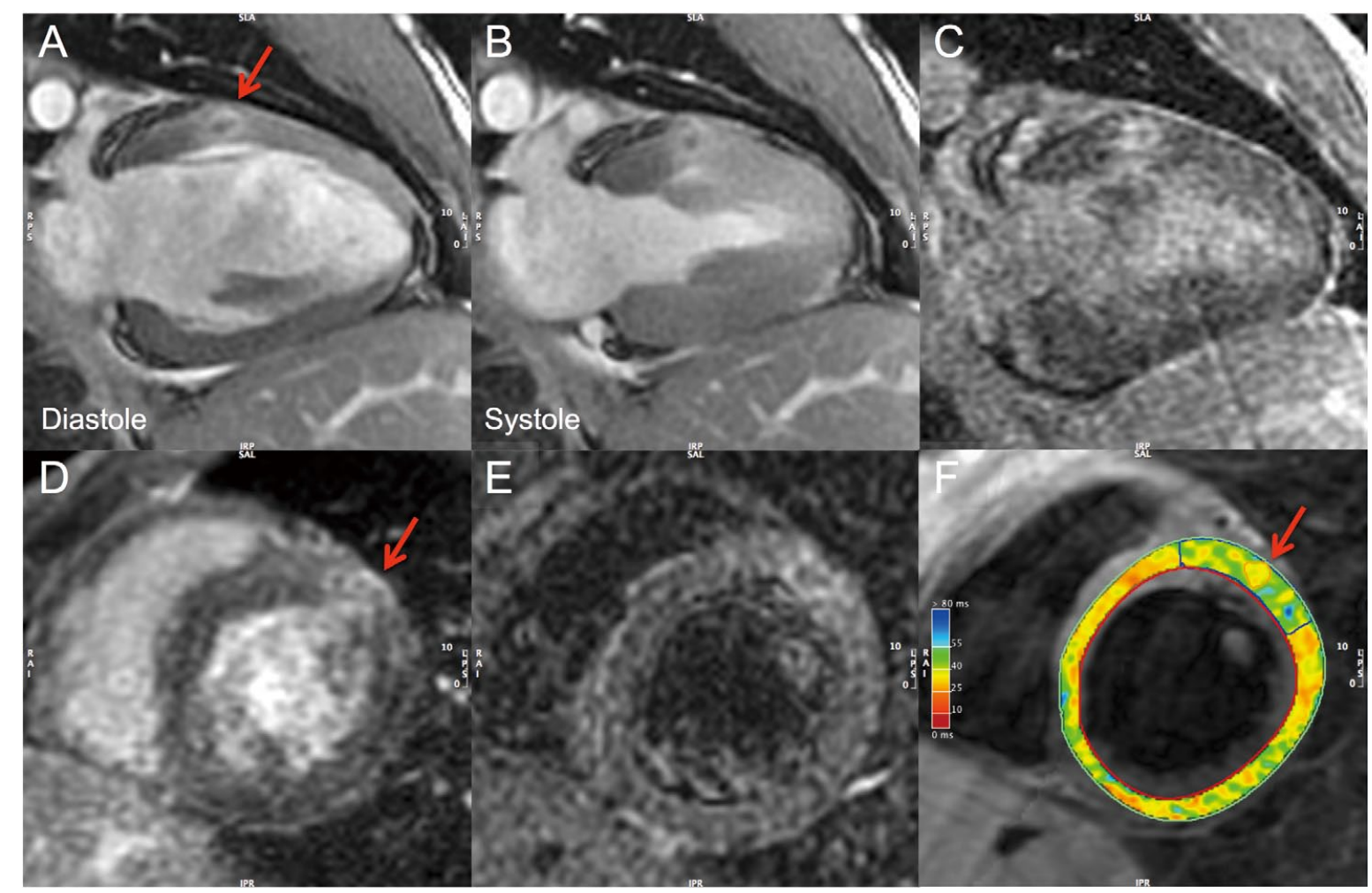

Figure. (A,B) Cine steady-state free-precession images acquired early after contrast showing focal myocardial hemorrhage in the basal anterior wall (arrow). (C,D) Late gadolinium enhancement (arrow). (E) T2-weighted image with an attenuated core, confirmed on (F) T2 mapping.

W e report the case of an 18-year-old man who presented following severe blunt chest trauma. This is one of the first cases demonstrating intramyocardial hemorrhage complicating myocardial contusion diag- nosed antemortum on cardiac magnetic resonance imaging (CMR), and highlights the potential role of CMR in myocardial trauma.

The man presented, following a high-speed motorbike ac-

Received June 3, 2014; revised manuscript received August 20, 2014; accepted September 1, 2014; released online October 2, 2014 Time for primary review: 28 days

Department of Cardiovascular Medicine (A.J.C.B., J.L.H., P.J.F., D.M.K., A.J.T.), Department of Trauma (M.F.), Intensive Care Unit (A.J.C.B., D.J.C., D.M.), Alfred Hospital, Melbourne, Victoria; Department of Epidemiology and Preventive Medicine, School of Public Health and Preventive Medicine, Monash University, Melbourne, Victoria (A.J.C.B., D.J.C., D.M.); and Baker IDI Heart and Diabetes Institute, Melbourne, Victoria (J.L.H., D.M.K., A.J.T.), Australia

Mailing address: Aidan J.C. Burrell, MD, Department of Cardiovascular Medicine, Alfred Hospital, 55 Commercial Road, Melbourne, Vic. 3181, Australia. E-mail: aidan.burrell@monash.edu

ISSN-1346-9843 doi:10.1253/circj.CJ-14-0626

All rights are reserved to the Japanese Circulation Society. For permissions, please e-mail: cj@j-circ.or.jp 
cident, with dyspnea, abdominal pain and sternal bruising. Electrocardiogram (ECG) showed transient hyperacute $\mathrm{T}$ waves in V1-V2; and troponin-I was 6,222 ng/L (normal, <26). Computed tomography (CT) showed a right-sided pneumothorax, normal mediastinum and a ruptured spleen. Transthoracic echocardiography was normal. CMR (Signa HD 1.5T; GE Healthcare, Waukesha, WI, USA) day 5 showed evidence of focal hypokinesia in the basal anterior wall (Figures A,B; Movie S1) in a non-coronary territory. Late gadolinium enhanced imaging showed this to be of high signal intensity but with an attenuated core (Figures C,D). Conventional T2-weighted imaging was performed using a short inversion time inversion recovery sequence (slice thickness, $8 \mathrm{~mm}$; repetition time, $2 \times \mathrm{R}-\mathrm{R}$ interval; echo time, $82 \mathrm{~ms}$; inversion time, $150 \mathrm{~ms}$ ). This showed marked regional increase in signal intensity with a central area of attenuation (Figure E), which was confirmed quantitatively on T2 mapping (multi-echo double-IR fast spin echo technique; GE Healthcare), demonstrating a regional segmental T2 time of $43 \mathrm{~ms}$ with a central area of attenuation of $37 \mathrm{~ms}$ vs. remote myocardial T2 $35 \mathrm{~ms}$ (Figure F). These findings are diagnostic of intramural hemorrhage. There were no significant cardiac sequelae observed, and the patient was discharged on day 11.

Cardiac contusion causing death occurs in up to $21 \%$ of patients who undergo autopsy following severe trauma. ${ }^{1}$ The diagnosis of the condition in patients who survive to hospital, however, has been challenging owing to the lack of a gold standard diagnostic test. ${ }^{2}$ Patients who are diagnosed with cardiac contusion can have symptoms that range from subclinical, through to malignant arrhythmias, heart failure and myocardial infarction (MI). ${ }^{3}$ Intramyocardial hemorrhage represents more extensive myocardial damage, and is associated with cardiac disruption, free rupture and death. ${ }^{4,5}$

There is growing interest in the use of CMR for the diagnosis of cardiac injuries in chest trauma. ${ }^{6}$ Compared to ECG, echocardiography and CT, CMR provides greater tissue characterization, and lack of distortion from adjacent rib or pulmonary trauma, making it ideal in severe chest trauma. It is, for example, capable of distinguishing cardiac contusion from acute MI, which may present similarly. ${ }^{7}$ In the current case, the focal midwall nature of the injury and the lack of a subendocardial pattern of necrosis within a coronary distribution made the differential diagnosis of MI very unlikely. In the present report, CMR allowed us not only to rule out $\mathrm{MI},{ }^{8}$ but also to diagnose the presence of myocardial hemorrhage antemortum, and when other modalities did not. CMR adds additional diagnostic information to currently used diagnostic modalities used in chest trauma, and it appears to have a promising but as yet undefined role in chest trauma.

\section{Disclosures}

Funds: This work was supported by an Alfred Hospital Small Project Grant. Industry: Dr Aidan Burrell is supported by a scholarship by Heart Foundation, Australia.

\section{References}

1. Turk EE, Tsang YW, Champaneri A, Pueschel K, Byard RW. Cardiac injuries in car occupants in fatal motor vehicle collisions: An autopsy-based study. J Forensic Leg Med 2010; 17: 339-343.

2. Clancy K, Velopulos C, Bilaniuk JW, Collier B, Crowley W, Kurek $\mathrm{S}$, et al. Screening for blunt cardiac injury: An Eastern Association for the Surgery of Trauma practice management guideline. J Trauma Acute Care Surg 2012; 73(5 Suppl 4): S301 -S306.

3. Maenza RL, Seaberg D, D'Amico F. A meta-analysis of blunt cardiac trauma: Ending myocardial confusion. Am J Emerg Med 1996; 14: $237-241$.

4. RuDusky BM. Myocardial contusion culminating in a ruptured pseudoaneurysm of the left ventricle: A case report. Angiology 2003; 54: $359-362$.

5. Hosaka Y, Kodama M, Chinushi M, Washizuka T, Sugiura H, Satou $\mathrm{K}$, et al. Images in cardiovascular medicine: Intramyocardial hemorrhage caused by myocardial contusion. Circulation 2004; 109: 277.

6. Moccetti M, Wyttenbach R, Santini P, Previsdomini M, Corti R, Gallino A. Images in cardiovascular medicine: Posttraumatic cardiac contrecoup: In vivo evidence by cardiac magnetic resonance imaging. Circulation 2009; 119: 1538-1540.

7. Baccouche H, Beck T, Maunz M, Fogarassy P, Beyer M. Cardiovascular magnetic resonance of myocardial infarction after blunt chest trauma: A heartbreaking soccer-shot. J Cardiovasc Magn Reson 2009; 11: 39 .

8. Nakamori S, Matsuoka K, Onishi K, Kurita T, Ichikawa Y, Nakajima $\mathrm{H}$, et al. Prevalence and signal characteristics of late gadolinium enhancement on contrast-enhanced magnetic resonance imaging in patients with takotsubo cardiomyopathy. Circ J 2012; 76: 914-921.

\section{Supplementary Files}

Supplementary File 1

Movie S1. Magnetic resonance imaging cine showing focal myocardial hemorrhage in the basal anterior wall of the left ventricle, with evidence of focal hypokinesia.

Please find supplementary file(s);

http://dx.doi.org/10.1253/circj.CJ-14-0626 